\title{
Spinocellular Penile Carcinoma
}

\author{
Gavrail Poterov, Georgi Tchernev* \\ Onkoderma-Clinic for Dermatology, Venereology and Dermatologic Surgery, General Skobelev, Sofia,Bulgaria
}

\begin{abstract}
Received: May 21, 2020 ; Accepted: May 25, 2020; Published: May 27, 2020
*Corresponding author: Professor Georgi Tchernev, PhD, Onkoderma-Clinic for Dermatology,Venereology and Dermatologic Surgery, Sofia, Bulgaria, Tel. No: 00359885588424; E-mail: georgi_tchernev@yahoo.de
\end{abstract}

\section{Case report}

We present a 50-year-old man with a complaint about the appearance of lesion located on the penis dating from 8-9 months(Figures 1a, 1b). The results of the outpatient cytological examination showedmorphological characteristics of suspected spinocellular carcinoma. Until the moment of hospitalization, the patient treatedtreated the lesion only topically with the following medications: Methylprednisoloneaceponate cream 0.1\%, Miconazole nitrate 2\% / Hydrocortisone 1\% cream and from four weeks has startedapplications of Imiquimod 5\% Cream. The patient was hospitalized for clinical clarification of the diagnosis and determination of the most appropriate therapeutic approach.

Within the dermatological examination it was established the presence of a lesion located on the entire surface of the glans penis, consisting of redheterogeneous, irregular mass formation, with a whitish deposit on its entire surface, with ulcerations and presence of a dark nodule(Figuress $1 \mathrm{a}, 1 \mathrm{~b}, 1 \mathrm{c}, 1 \mathrm{~d}$ ). From the conducted paraclinical tests, no data on inflammation was found. The ultrasound and radiography of the lungs and abdomen showed no pathological changes.A screening for metastasis was performed with no
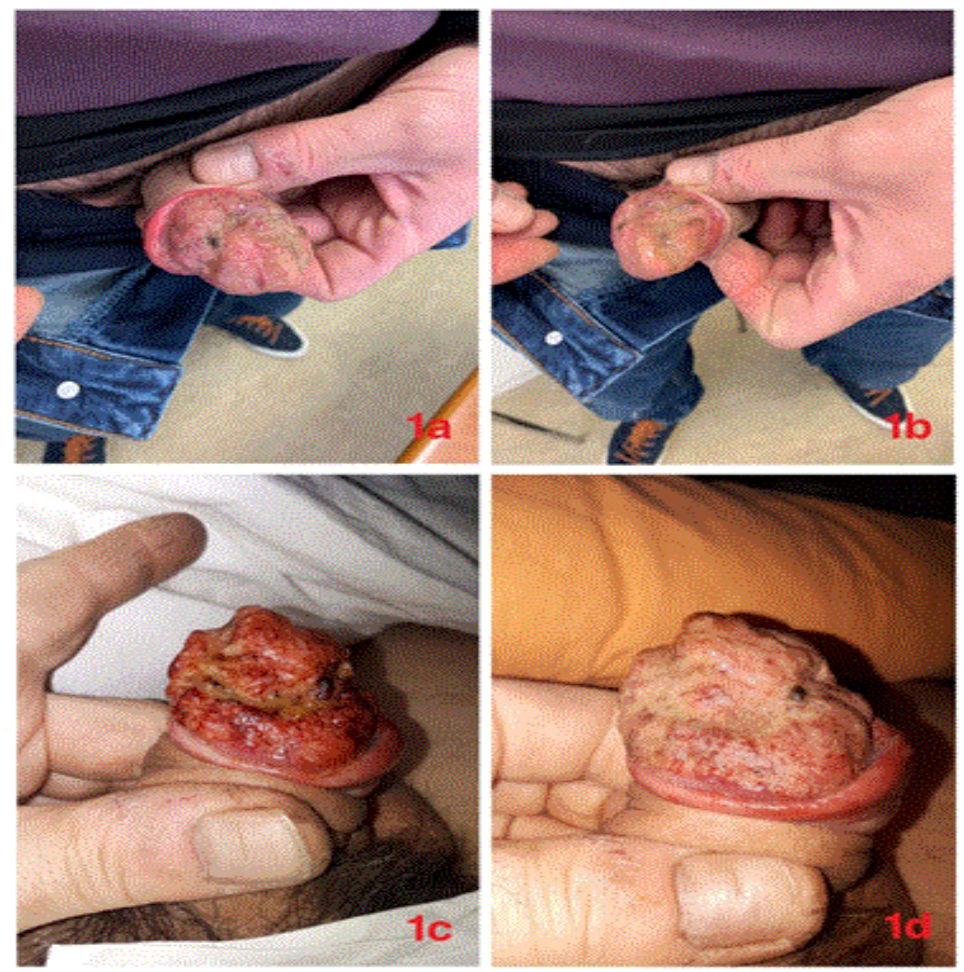

Figure 1a,1b: Showsa lesion located on the glans penis, consisting of heterogeneous, irregular mass formation, with partial whitish deposit on its surface, ulcerations and presence of a dark nodule.

Figure 1c, 1d: Show the progression of the lesion, consisting of heterogeneous, irregular mass formation with a whitish deposit on its entire surface and deepening of the ulcerations. 
evidence for a metastatic disease.From the collected anamnestic and available clinical data, we concluded that the probable diagnosis isa highly aggressive spinocellular carcinoma.A biopsy of the lesion was planned to be taken to prove the diagnosis, as well as performing of subsequent penectomy in order to increase survival rate.Spinocellular carcinoma (Squamous Cell Carcinoma) (SCC) of the penis is very uncommon disease. It is thought to affect patients between 50-60 yrs. The most important risk factors for its development are the poor personal hygiene, uncircumcision, HPV, chronic inflammatory skin conditions, such as Lichen Sclerosus. The pathogenesis of this disease is not fully understood. Poor hygiene contributes to the development of the tumor through the accumulation of smegma and other irritants in the balanoprepulative sulcus, and also the increased risk of bacterial and candidal infections [1]. Uncircumcision is also thought to increase the risk of its development [2]. There are two different clinical and histological forms of SCC of the penis (carcinoma in situ and invasive carcinoma), each with different prognostic and therapeutic effects. SCC in situ arises from a precursor lesion, which can be classified according to the severity and degree of cellular atypia and the presence or absence of HPV infection [3]. Clinically, SCC in situ is quite variable - from subclinical lesions to red lesions: Bowen's genital disease, leukoplakia, and Bowenoidpapulosis.Clinically, the invasive SCC of the penis is highly variable, ranging from an erythematous plaque or ulcer to an exophytic or verruciform tumor. The size of the lesions can be up to a few inches in diameter and can bleed as well.For the diagnosis a biopsy and subsequent histopathological examination are very important. Spinocellular carcinoma of the penis in a differential diagnostic aspect should be distinguished from: Bowen Disease of the Glans Penis, VerruciformXanthoma, Verrucous Carcinoma, Warts.

The treatment is specific and varies according to the clinical stages of the disease. It includes: radiation therapy, topical (imiquimod 5\% cream) and systemic drug therapy and surgery. Penile SCC in situ can be treated by simple surgical excision or Mohs micrographic surgery [4],which increases the chance for preserving penile function and reducing the risk of recurrence. The main treatment for invasive SCC of the penis is surgical resection of the primary tumor with a range of 5 to $10 \mathrm{~mm}$. The purpose of treatment is fully to eliminate the disease and, where possible, preserve urinary and sexual function [5]. Surgical dissection of the lymph nodes is also recommended only if they are enlarged and do not regress after adequate antibiotic therapy.

In conclusion, the early and correct diagnosis of the initial lesions, as well as subsequent treatment of spinocellular carcinoma of the penis, may increase the survival rate and quality of life of the patients.

\section{Authors' contributions:}

We all provided clinical care for the patient, wrote and approved the manuscript.

\section{Declaration of interest:}

We declare no competing interests.

\section{References}

1. RS Van Howe, FM Hodges.The carcinogenicity of smegma: debunking a myth.J EurAcad Dermatol Venereol. 2006;20(9):1046-1054.

2. JR Daling, MM Madeleine, LG Johnson, SM Schwartz, KA Shera, MA Wurscher, et al. Penile cancer: importance of circumcision, human papillomavirus and smoking in in situ and invasive disease. Int J Cancer. 2005;116(4):606-616.

3. EJ Schoen, M Oehrli, C Colby, G Machin.The highly protective effect of newborn circumcision against invasive penile cancer.Pediatrics. 2000;105(3):E36.

4. AW Shindel, MW Mann, RY Lev, R Sengelmann, J Petersen, GJ Hruza, et al.Mohs micrographic surgery for penile cancer: Management and long-term followup.J Urol. 2007;178(5):1980-1985.

5. CPulidoa, I Torresb, VPatosa.Penile Squamous Cell Carcinoma. ActasD ermosifiliogr.2012;103(6):478-487. 